\title{
THEORY OF FORBUSH DECREASE IN A MAGNETIC CLOUD
}

\section{Anastasia Petukhova ${ }^{1}$}

Yu.G. Shafer Institute of Cosmophysical Research and Aeronomy, SB RAS, Yakutsk, Russia 31 Lenin Ave., Yakutsk, Russia

E-mail: petukhova@ikfia.ysn.ru

\section{Ivan Petukhov}

Yu.G. Shafer Institute of Cosmophysical Research and Aeronomy, SB RAS, Yakutsk, Russia 31 Lenin Ave., Yakutsk, Russia

E-mail: i vaneikfia.ysn.ru

\section{Stanislav Petukhov}

Yu.G. Shafer Institute of Cosmophysical Research and Aeronomy, SB RAS, Yakutsk, Russia 31 Lenin Ave., Yakutsk, Russia

E-mail: petukhov@ikfia.ysn.ru

A model of Forbush-decrease in a magnetic cloud is presented. We calculate moments of the particle distribution function depending on time. The calculated results of cosmic ray intensity generally agree with the observed ones in events registered by ground based detectors. It is found that the magnetic flux rope is of great importance in dynamics of Forbush decrease. 


\section{Introduction}

Coronal mass ejections (CMEs) affect near-Earth space. They can include magnetic clouds (MCs), which have the strongest influence on Forbush decreases (FD). Theoretical studies of the spatial distribution of cosmic rays (CRs) are based on the solution of the diffusive transport equation. MC is presented as an infinitely extended cylinder with a constant radius moving in interplanetary space in [1], the expansion of the cylinder is taken into account in [2]. However, the cylindrical model has its shortcomings as spatial distribution of cosmic rays (CR) depends on magnetic flux rope (MFR) due to their high mobility. Thus, the toroidal model is more appropriate for these studies $[3,4]$.

We present a MFR model in a MC, a method for calculating the CR distribution function in a MC, and the relations with its three moments. The calculation results and the measurements are compared.

\section{MC model and calculation method}

We suggest that a MC having the shape of a torus segment is located inside a CME with a fixed distribution of the radial velocity at the initial moment. The lateral boundaries of the segment will be referred to as ends. The initial MFR is described by the solution of Miller and Turner [5]. The subsequent MC motion is determined by the kinematic model, which describes the velocity conservation of the Lagrangian particles in a CME. The MFR during its motion is determined by the freezing-in condition represented as the conservation of the magnetic flux through the areas associated with the Lagrangian particle flow. The calculation of the particle distribution function is based on the Liouville theorem: the density of the particle distribution function retains along their trajectories, which are characteristics of the Boltzmann equation. We assume that: 1) Parker's solar wind is outside the MC; 2) the density of the distribution function is isotropic and homogeneous in the region ahead of $\mathrm{MC}$.

The model MC is a segment of the magnetic cloud. Real MCs are connected to the Sun by means of magnetic field lines and the model MC describes their central parts. The MFR (the field direction changes from perpendicular on the surface to longitudinal at the center of the cross-section) determines the particle propagation. As a result, particles from the surrounding space fill only the near surface region of the cloud segment. A significant part of the particles comes to its ends. The particles can fill the near axis region when they return to the segment after redistribution along the field lines in the regions connecting the MC to the Sun. We introduce the loss factor of particles at the ends of $\alpha=\left(I_{1}-I_{2}\right) / I_{1}$, which is a free parameter. Here $I_{1}, I_{2}$ are the particle fluxes coming to the ends and returning back to the segment. It is also assumed that the distribution function of the returning particles is isotropic and homogeneous over the cross-section of the end. The accepted assumptions are sufficient to calculate the particle distribution function.

In theory, the particle distribution function is calculated but in experimental studies the moments of the distribution function are determined. To obtain the relations between the distribution function and its moments, we write the relation

$$
f(\vec{p})=\sum_{i} n_{i} b_{i}+\sum_{i, j} n_{i} n_{j} f_{i j}
$$

where $f$ is distribution function; $n_{i}=p_{i} / p$ is the unit vector of the particle motion direction; $p_{i}$, $b_{i}$ are the momentum and vector components, respectively; $f_{i j}$ is the symmetric 2 nd-order 
tensor. The components of the unit vector is defined by two angles. We determine the components of the vector and six components of the tensor by multiplying (1) by various trigonometric functions of these angles and integrating over the solid angle. For 2 moments we obtain

$$
f_{0}=\int f d \Omega / 4 \pi=\left(f_{11}+f_{22}+f_{33}\right) / 3, \vec{A}=-\left(\vec{b} / f_{0}\right) 100 \%,
$$

where $f_{0}$ is the distribution function density averaged over the solid angle ( the zero moment), $\vec{A}$ is anisotropy (the first moment), and $d \Omega$ is the differential of the solid angle.

Any symmetric 2nd-order tensor can be reduced to the diagonal form. For this purpose, we calculate the eigenvalues and eigenvectors of the tensor. The diagonal form of the tensor corresponds to the ellipsoidal form of the angular dependence of the distribution function referred to as the second moment. The ellipsoid shape depends on the ratio of the eigenvalues, with its orientation being determined by the eigenvectors.

\section{Calculation results and discussion}

We used the following parameters of the initial torus segment in the demonstration calculation: the torus cross-section radius is $0.043 r_{e}\left(r_{e}\right.$ is the astronomical unit), the torus axis is located in the solar equator plane at $0.457 r_{e}$ from the Sun; angular width of the segment is $\pi / 2$; the magnetic field intensity on the torus axis is $20 \mathrm{nT}$; MFR is SWN-type; the torus expansion during its radial motion is produced by a spherically symmetric CME, the velocities of the Lagrangian particles of which are distributed linearly along the radius from 500 to 400 $\mathrm{km} / \mathrm{s}$ within the torus. MC is surrounded by the Parker's solar wind. The interaction between the MC and the solar wind is not taken into account. The parameters shown in the figures refer to the point with the coordinates of $x=1 r_{e}, y=z=0$ in the heliocentric Cartesian coordinate system. The chosen point is located in the plane dividing the torus segment into equal parts. Time step is 1 hour. Kinetic energy of particles is $\varepsilon_{k}=10 \mathrm{GeV}$. Figure 1 shows the magnetic field magnitude and its components in the MC in time. As can be seen from the figure, the chosen point is located inside the MC in the interval from 42 to 61 hours. The maximum value of the magnetic field is $20 \mathrm{nT}$, it did not change during the $\mathrm{MC}$ propagation.

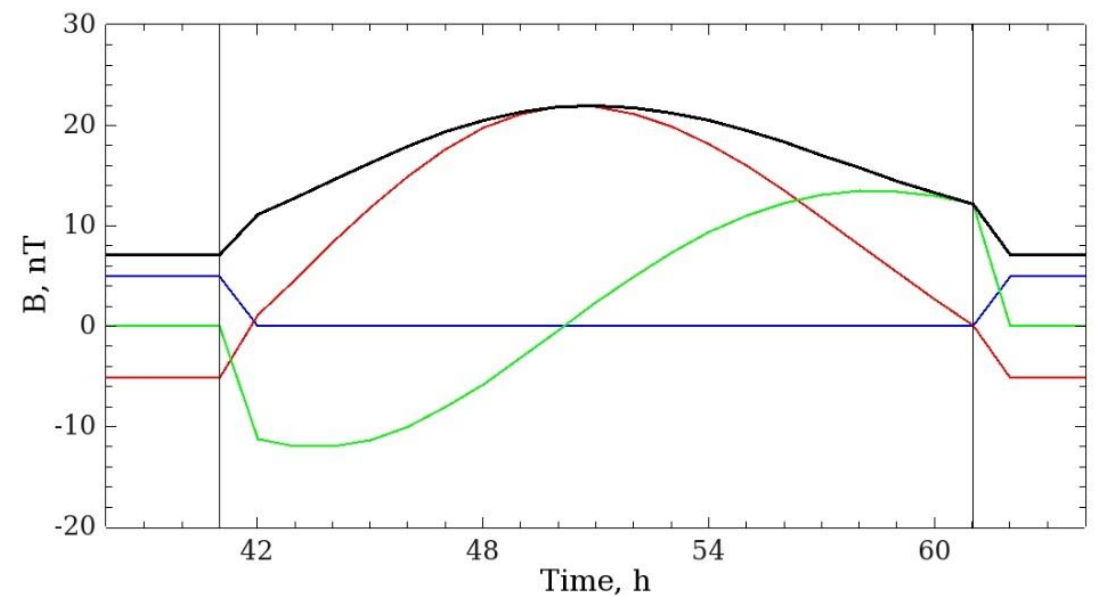

Figure 1: Magnetic field magnitude and its components: black line is vector magnitude, blue line is $B_{x}$, red line is $B_{y}$, green line is $B_{z}$. The vertical lines are MC boundaries. 
Figure 2 shows the FD amplitude of $A_{F D}=\left(\left(f_{0}-f_{00}\right) / f_{00}\right) 100 \%$, where $f_{00}$ is the distribution function density outside the MC. The curves from top to bottom correspond to the values set of $\alpha=(0-5) \%$. The maximum value of $A_{F D}$ changes from 0.2 to $2.5 \%$. Thus, the greater the loss factor at the segment ends, the greater $A_{F D}$.

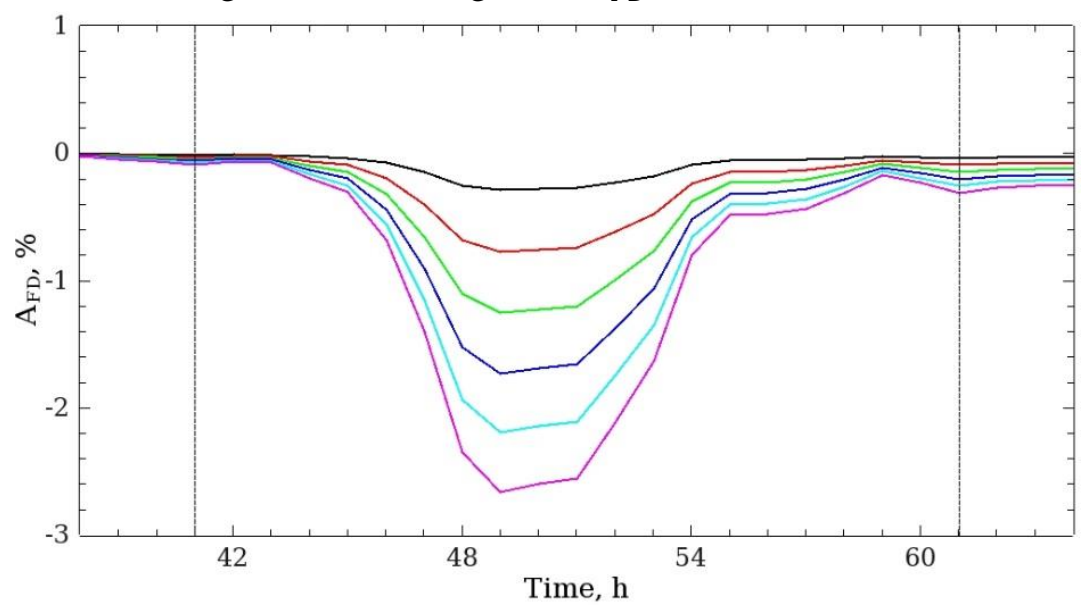

Figure 2: Amplitude of FD in time. The curves from top to bottom correspond to $\alpha=$ $(0-5) \%$. The vertical lines are MC boundaries.

Figures 3, 4 show the anisotropy components with $\alpha=5 \%$ as a time function for two MFR types: SWN type in Figure 3 and SEN type in Figure 4. Each component has a feature in temporal behavior. Component $A_{x}$ is the smallest, components $A_{y}, A_{z}$ change their sign two and one time crossing the $\mathrm{MC}$, respectively. One can see stable dependences in the behavior of the anisotropy components. In the region with the maximum values of component $B_{y}$ (interval (4855) hours according to Figure 1), $A_{y}$ has a minus sign. For component $A_{z}$, the $\mathrm{MC}$ crossing time can be divided into two intervals: the first interval starts from the entrance into the MC and ends at its center; the second one starts from the center and ends at the exit from the MC. The component sign in the first interval depends on the sign of component $B_{y}: A_{z}>0$ when $B_{y}>0$ and vice versa. The boundary between the intervals coincides with the sign change of component $B_{z}$, i.e. in the vicinity of the magnetic cloud center. For NWS and NES types $A_{y}$ has a plus sign. Sign of component $A_{z}$ in the first interval depends on the sign of component $B_{y}$ : $A_{z}>0$ when $B_{y}>0$ and vice versa, as well as for SWN and SEN types. These relations confirm the possibility to determine the magnetic field properties based on measuring the $\mathrm{CR}$ intensity by ground-based detectors [6]. 


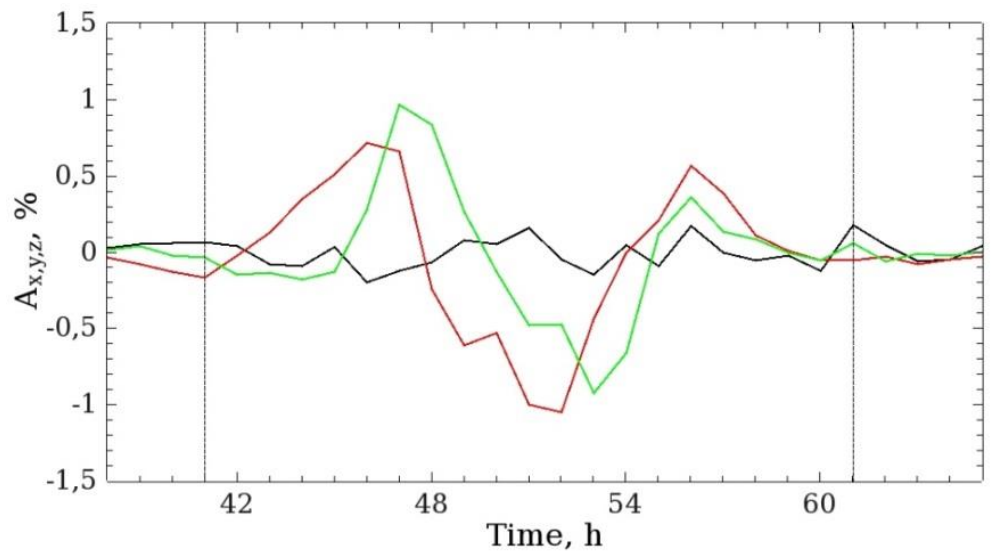

Figure 3: Anisotropy components with $\alpha=5 \%$ in time: black line is component $A_{x}$, red line is $A_{y}$, green line is $A_{z}$. SWN type of the MC.

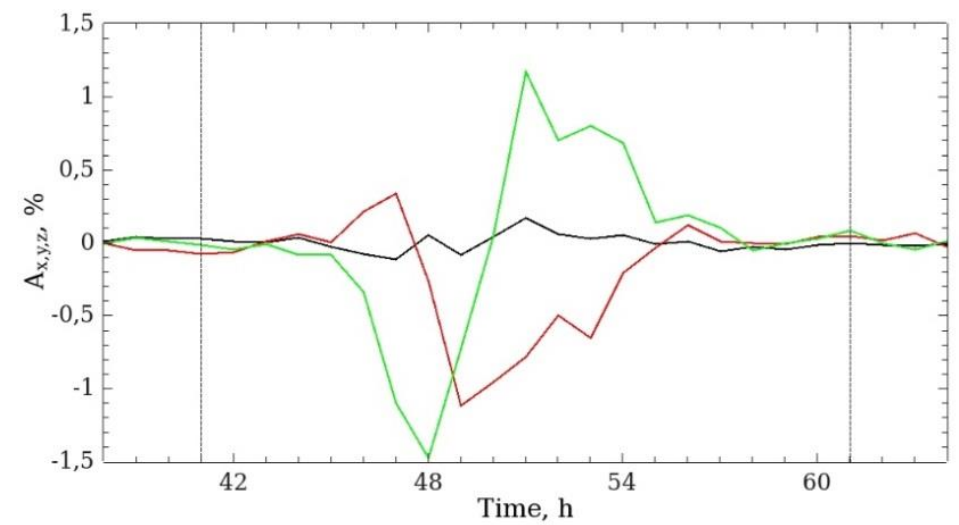

Figure 4: Anisotropy components with $\alpha=5 \%$ in time: black line is component $A_{x}$, red line is $A_{y}$, green line is $A_{z}$. SEN type of the MC.

The anisotropy magnitude depends on the loss factor $\alpha$ at the ends of the magnetic cloud segment. Figure 5 shows the anisotropy components depending on time for different $\alpha$. The components with $\alpha=5$ coincide with the model ones shown in Figure 3. As can be seen, the behavior of the anisotropy components does not depend on $\alpha$ in time, however, the anisotropy amplitude shows a dependence: the smaller $\alpha$, the less the anisotropy, as well as the FD amplitude.

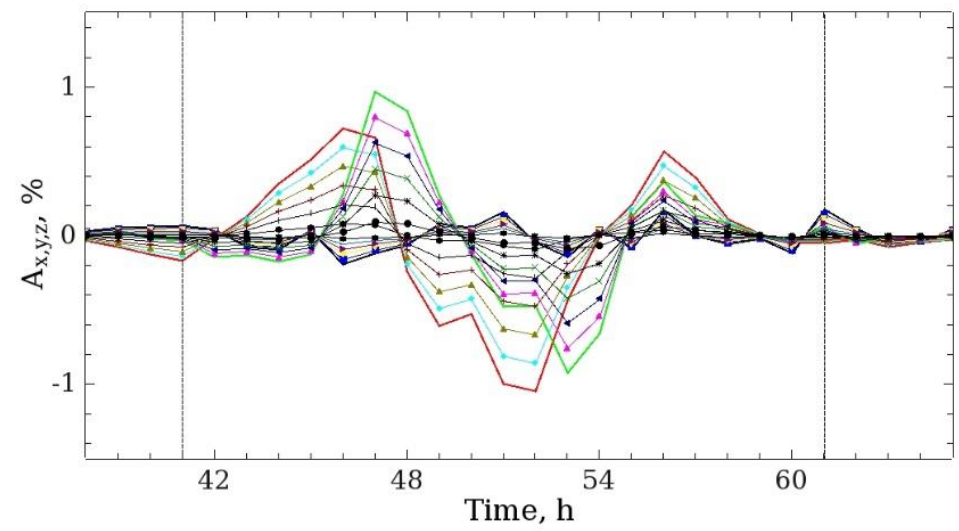

Figure 5: Anisotropy components with $\alpha=(0-5) \%$ in time. SWN type of the MC. 
Figure 6 illustrates the equatorial component of anisotropy in the form of vector sum of hourly values of $A_{x}, A_{y}$, which are the anisotropy components. As can be seen from the Figure 6: 1) the anisotropy significantly increases in the MC (43-57) hours; 2) component $A_{y}$ is significantly higher than component $\left.A_{x} ; 3\right)$ component $A_{y}$ increases within (42-47) hours and decreases within (47-54) hours; 4) the anisotropy vector rotates: the rotation is anticlockwise in the interval during (45-51) hours and clockwise during (51-56) hours.

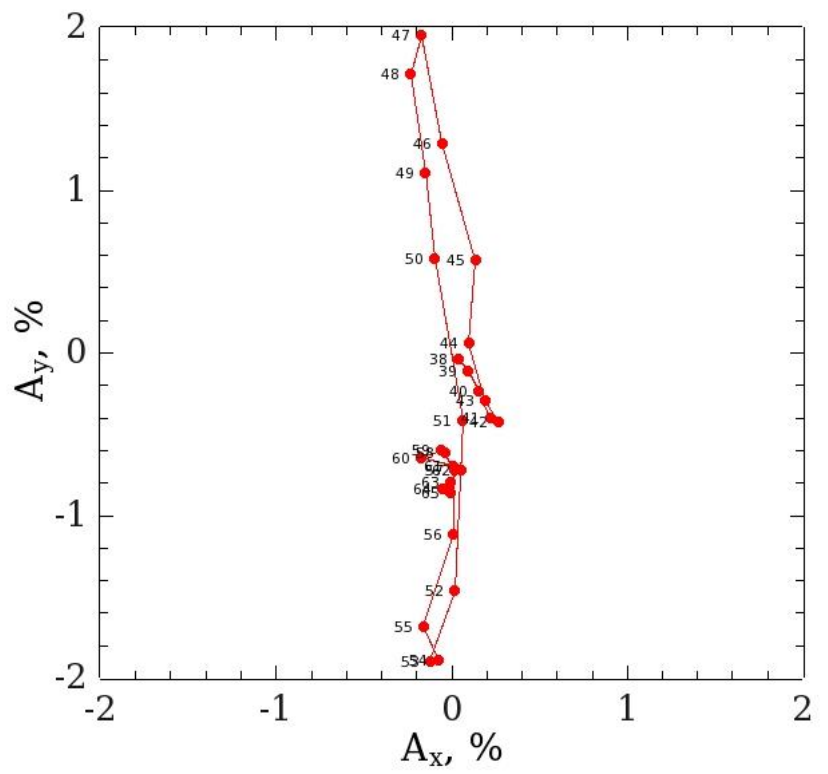

Figure 6: Equatorial component $A_{x, y}$ of the anysotropy. SWN type of the MC.

Figures 7,8 show the eigenvalues and the orientation angles of the second moment of the CR distribution function. As can be seen from Figure 7, the difference between two eigenvalues is $\sim 0.3 \%$, and their difference from the third eigenvalue is $\sim 3 \%$ in the interval (46-54) hours. Outside the interval, the eigenvalues coincide with the accuracy of $\sim 0.3 \%$. Thus, the second moment of the CR distribution function in the interval (46-54) hours represents a biaxial ellipsoid, in which the smallest of the axes differs from the other two by $\sim 3 \%$. Outside this time interval, the second moment represents the sphere with the accuracy of $\sim 0.3 \%$. Comparing Figures 2 and 7, it is clear that the ellipsoid of the second moment coincides with the FD in time.

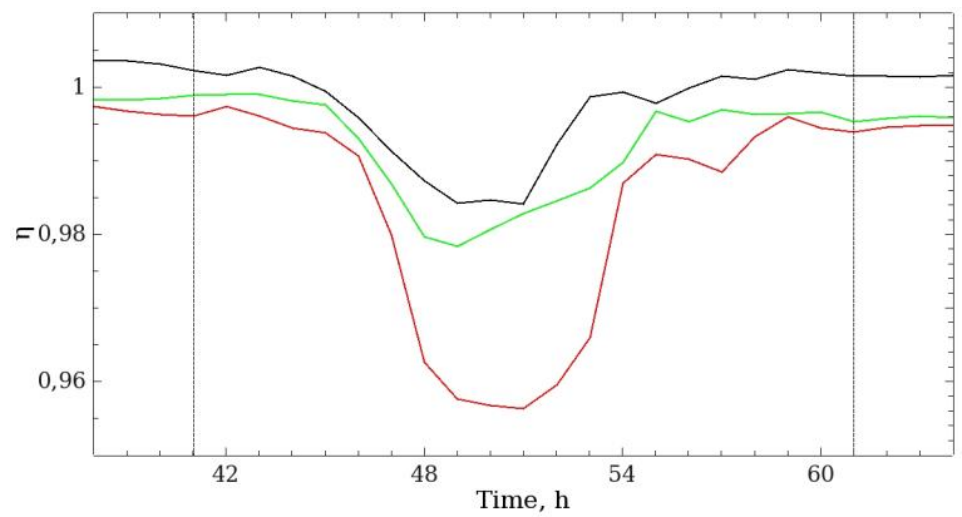

Figure 7: Eigenvalues of the second moment of the CR distribution function in time. 


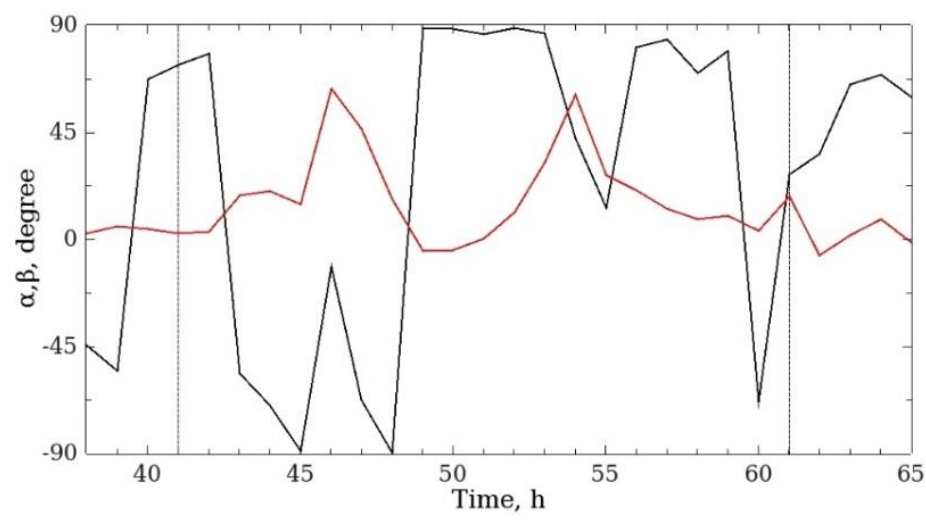

Figure 8: Orientation angles of the second moment of the CR distribution function in time. Black curve is course angle and red one is tangage angle.

The orientation of the ellipsoid in space is determined by 3 Euler angles: course, tangage and bank. The orientation of a biaxial ellipsoid is determined by 2 angles: $\alpha$ is course angle - the angle between the projection of the smallest axis on the plane $\mathrm{X} 0 \mathrm{Y}$ and the $\mathrm{X}$ axis; $\mathrm{B}$ is tangage angle - the angle between this projection and the $\mathrm{Z}$ axis.

As can be seen from Figure 8, in the interval (48-54) hour, $\alpha \simeq \pi / 2$ (as for the ellipsoid axis $\alpha=-\pi / 2=\pi / 2$ ) and $\beta$ monotonically changes from $\pi / 4$ to 0 and back to $\pi / 4$.

The calculation of the CR distribution function with $60 \mathrm{GeV}$ energy in the MC with similar parameters showed the absence of FD. It reveals that the FD amplitude depends on the magnetic field strength and the MC cross-section radius. The presented results are obtained for a $\mathrm{MC}$, whose axis is located in the solar equator plane. Comparing the calculation results with the measurements, it is necessary to take into account the trajectory of the MC crossing, on which the time dynamics of the moments of the CR distribution function significantly depends.

The work [7] presents the results of the analysis of the CR density and anisotropy with the rigidity of $10 \mathrm{GV}(9 \mathrm{GeV})$ in 99 events of the 23rd and 24th solar cycles, in which the presence of MCs is confirmed.

In the studied sample, there is a great diversity in: motion velocity, the gradient of the flow velocity within the MC, the distribution and magnetic field magnitude in the MC. The time dynamics of the CR density and anisotropy of is also diverse. In this sample, the characteristic behavior of density and anisotropy in MCs is presented: 1. the maximum value of $A_{F D}$ is within $(1-12) \%$; 2. the density drop is sharp, and the recovery is more gradual; 3. the anisotropy changes significantly at the entrance and/or exit from the MC; 4. monotonic change of the anisotropy is often observed inside the MC; 5 . a rotation of the equatorial component $A_{x, y}$ is possible; 6 . the north-south component $\left(A_{z}\right)$ often changes its sign in the vicinity of the MC center. As can be seen from the results presented in Figures 2-6, the model calculations generally coincide with the observed behavior of the density and anisotropy in the MC. 


\section{Conclusions}

1. We show a model of a dynamic MC located in an inhomogeneous matter flow. A method for calculating the MFR in the MC initially being a torus segment is presented and applied.

2. A method for calculating the particle distribution function in the MC using particle trajectories is presented and applied.

3. The relations between the particle distribution function and its 3 moments are obtained.

4. The influence of the regions connecting MC with the Sun on the CR distribution in the MC is determined. This effect is due to the MFR.

5. Time dynamics of 3 moments of the particle distribution function in the $\mathrm{MC}$ is calculated. The dynamics of the CR density and anisotropy generally agree with the measurements.

6. The found relations between the dynamics of the distribution function moments and the MFR confirm the possibility to determine the magnetic field properties based on measuring the CR intensity by ground-based detectors.

The work is supported by Russian Foundation for Basic Research (Project №15-4205085-р_восток_a).

\section{References}

1. H.V. Cane, I.G. Richardson, and G. Wibberenz, The Response of Energetic Particles to the Presence of Ejecta Material, Proc. 24th Int. Cosmic Ray Conf. (1995) 4: 377-380.

2. K. Munakata, S. Yasue, C. Kato, et al., On the cross-field diffusion of galactic cosmic rays into an ICME, Adv. Geosci. (2006) 2: 115.

3. A.S. Petukhova, I.S. Petukhov \& S.I. Petukhov, Forbush decrease in the intensity of cosmic rays in a toroidal model of a magnetic cloud, Jetp Lett. (2015) 102: 697.

4. A.S. Petukhova, I.S. Petukhov \& S.I. Petukhov, V. G. Grigoryev, Forbush decrease in the intensity of cosmic rays in a toroidal model of a magnetic cloud, Bull. Russ. Acad. Sci. Phys. (2017) 81: 532.

5. G. Miller, L. Turner, Force free equilibria in toroidal geometry. Phys. Fluids. (1981) 24: $363-$ 365 .

6. T. Kuwabara, J. W. Bieber, P. Evenson, et al., Determination of interplanetary coronal mass ejection geometry and orientation from ground-based observations of galactic cosmic rays, J. Geophys. Res. (2009) 114(A8): A05109.

7. A. Abunin, M. Abunina, A. Belov, et al. The impact of magnetic clouds on the density and the first harmonic of the cosmic ray anisotropy, Proc. 33-rd Int. Cosmic Ray Conf. (2013). ID 198. 